\title{
Consider Embracing the Reviews from Physician Rating Websites
}

\author{
Howard S. Gordon, MD
}

Jesse Brown Veterans Affairs (VA) Medical Center and VA Center of Innovation for Complex Chronic Healthcare, and Section of Academic Internal Medicine, Department of Medicine, University of Illinois at Chicago College of Medicine, Chicago, IL, USA.

J Gen Intern Med 32(6):599-600

DOI: $10.1007 / \mathrm{s} 11606-017-4036-8$

(c) Society of General Internal Medicine 2017

$I^{n}$ $\mathrm{n}$ an era of social media use, with star rankings of many services, it is important to understand how patients and physicians view and use physician rating websites. Patients seeking health care increasingly use websites that contain patient ratings of physicians. These physician rating websites are a valuable source of information for patients seeking care and thus evaluation of perceptions about these websites is an important area of inquiry. Physician rating websites may provide several different types of information about physicians, including listings with information on training, specialty, certification, location, and availability of appointments, and may also contain patients' ratings of physicians from various patient survey measures.

\section{HOW DO PATIENTS AND PHYSICIANS PERCEIVE THE RATINGS AND COMMENTS ON PHYSICIAN WEBSITES?}

Evaluating the information posted on physician ratings websites can be a challenge. Website content may be the responsibility of the health system where the physician practices or an entity that is not connected to the practice or health system. It can be difficult to identify whether sites are reputable and whether they have profit motives or conflicts of interest that lead them to grant particular physicians or provider groups advantages in terms of placement or visibility. Information on the websites can vary substantially and may be limited in several ways. Not all physicians have listings and not all physicians listed have patient ratings. The number of ratings for each physician is often too low to provide reliable and meaningful assessments. In such cases, a positive or negative rating or review may be unduly influential. The source of the ratings is a potential concern for both patients and physicians. Because reviews may be anonymous, it can be difficult to verify who provided the ratings. Ratings may not be independent (e.g., physician staff may provide reviews), may not be offered by actual patients of the rated physician, and may not focus on the same outcomes (e.g., access rather than quality).

Published online March 15, 2017
Most online reviews of physicians are positive, with narrative comments tending to be more positive than negative and numerical ratings more mixed. ${ }^{1}$ The provision of rankings (e.g., star ratings) may be interpreted to imply that physician rating websites measure quality. Thus an important question to explore is whether physician rating website star ratings have validity for assessing the quality of care. Physician rating websites may not indicate the specific method used to produce quantitative ratings, although some clearly indicate that they use measures associated with quality of care (e.g., Physician Compare). Few studies have evaluated the association between star ratings and quality. Evidence of a significant association between posted ratings and patient experience quality measures has been reported, but the results were marginal. ${ }^{2}$ For a 20\% change in physician rating, the change in the patient experience quality measure was 0.8 to $1.7 \%$. Associations between physician ratings and clinical quality measures were not significant. ${ }^{2}$ Still, there are substantial methodological limitations in using physician rating website data for quality assessment, such as whether the data collection methods involve the use of validated measures and whether appropriate risk adjustment is applied.

Narrative comments on physician rating websites are qualitative assessments that tend to focus on the physician, staff, or practice, and may be global (i.e., communicating general impressions) or specific (i.e., focusing on particular aspects of care or service). ${ }^{1,3}$ Compared to numerical ratings, narrative ratings may receive a disproportionate share of attention. Numerical or star ratings can be summarized with a mean score, but narrative comments are individually displayed and draw the reader's gaze. Although the content of narrative comments may be qualitatively rich, such comments are less representative of the physicians' patients than are numerical ratings, in part because they are less frequent and are dependent on which patients choose to provide narrative comments. Nonetheless, narrative comments that are specific differ from numerical ratings in that they may provide valuable feedback that could be used for improvement (e.g., comments that focus on waiting time or patient perception of physicians' communication).

In this issue of JGIM, Halliday et al. ${ }^{4}$ report the results of a survey assessing physician and patient perceptions of independent physician rating websites and about publicly publishing health system-based patient experience surveys. The study provides the opportunity to compare patient and physician responses regarding making physician ratings publicly 
available and to compare views on websites with different ownership and potentially different standards of data collection. These authors found that viewing a physician rating website was common among half of the physicians and almost half the patients surveyed. Younger age among both patients and physicians was associated with visiting one of these websites. Physicians who worked in an ambulatory care clinic were more than twice as likely to have reported visiting a physician rating website. Patients who were women, had regular Internet access, and had a college education were more likely to visit these websites. Perhaps not surprisingly, patient and physician views were discordant on several measures. Physicians were more likely to trust patient experience surveys issued by health systems, whereas patients were more likely to trust ratings found on independent websites. Nonetheless, patients were more likely to support public release of health system patient experience survey results, while physicians were less likely to support making these data publicly available for patient viewing. Given some concern among physicians about narrative comments, it is interesting that there was no statistically significant difference in support for sharing narrative comments compared with numerical ratings.

\section{PHYSICIANS SHOULD CONSIDER HOW TO EMBRACE ONLINE REVIEWS}

At first look, the potential challenges of interpreting data on these websites has negatively influenced both patients' and physicians' opinions about website content. Patients may worry about whether the information is trustworthy, while physicians may be concerned about how bad reviews may tarnish their professional reputation. Privacy concerns might prevent patients from posting reviews, and privacy laws might prevent physicians from responding to bad reviews, because they must avoid disclosing details about a patient's care. The response to the rise in the popularity of physician rating websites is evolving, with early recommendations urging physicians to be patient-centered, recognize the value of understanding the patient's perspective, and use the ratings as feedback about areas in need of improvement. ${ }^{5}$ An impetus for the survey designed by Halliday et al. ${ }^{4}$ was the efforts by health systems to increase the accessibility of their internal patient experience surveys by making ratings derived from these surveys publicly available. Thus, these findings should be considered in the context of recent moves by some health systems to post survey results on their websites. Given the nature of the findings, many physicians might still be concerned with this level of transparency. However, the results of efforts for full transparency in health system surveys have been reported to be rewarding. The University of Utah Health Care system claims that their own efforts to survey all their patients and to publish all patient comments online have led to steady gains in quality metrics, a reduction in costs, and substantial improvements in patient ratings. ${ }^{6}$ Sourcing reviews on a health system website has potential advantages such as larger numbers of reviews per physician, the use of validated patient experience measures, and the ability to verify that reviews are from real patients (similar to Amazon.com verifying that reviews are from an actual customer), all of which could improve trust in the physician ratings. These advantages address some of challenges that have negatively influenced patients' and physicians' opinions about physician rating websites. A remaining barrier for some patients is the digital divide, where patients without regular Internet access are less likely to use rating websites. Diminished online access not only impedes patients' ability to gain valuable knowledge about health care organizations, but also prevents them from supplying feedback. Nonetheless, given the potential benefits and positive results from making patient experience survey findings publicly available, both patients and physicians stand to gain from the increased transparency provided by sharing patient experience survey results on physician rating websites. It's time for physicians to take another look at physician rating websites, to reconsider the benefits these websites offer in terms of using patient feedback to better understand patient needs and wants, and to then act on that information to make health care more patient-centered.

Corresponding Author: Howard S. Gordon, MD; Jesse Brown Veterans Affairs (VA) Medical Center and VA Center of Innovation for Complex Chronic Healthcare, and Section of Academic Internal Medicine, Department of MedicineUniversity of Illinois at Chicago College of Medicine, Chicago, IL, USA (e-mail: hsg@uic.edu).

\section{Compliance with Ethical Standards:}

Funding: This work was supported in part by grants \#CIN 13-415 and \#IIR 12-050 from the Department of Veterans Affairs, Office of Research and Development, Health Services Research and Development Service.

Notes: The views expressed in the article are those of the authors and do not necessarily represent the views of the Department of Veterans Affairs.

Conflict of Interest: Dr. Gordon has no relevant conflict of interest.

\section{REFERENCES}

1. Lopez A, Detz A, Ratanawongsa N, Sarkar U. What patients say about their doctors online: a qualitative content analysis. J Gen Intern Med. 2012;27(6):685-92.

2. Gray BM, Vandergrift JL, Gao GG, McCullough JS, Lipner RS. Website ratings of physicians and their quality of care. JAMA Intern Med. 2015; 175(2):291-3.

3. Emmert M, Meier F, Heider AK, Durr C, Sander U. What do patients say about their physicians? An analysis of 3000 narrative comments posted on a German physician rating website. Health Policy. 2014;118(1):66-73.

4. Halliday AM, Kachalia A, Meyer GS, Sequist TD. Physician and patient views on public physician rating websites. J Gen Intern Med. 2017. doi: 10. 1007/s11606-017-3982-5.

5. Jain S. Googling ourselves-what physicians can learn from online rating sites. N Engl J Med. 2010;362(1):6-7.

6 Lee VS. Why doctors shouldn't be afraid of online reviews. Last accessed February 10, 2017. Available from: https://hbr.org/2016/03/why-doctors-shouldnt-be-afraid-of-online-reviews /. 